\title{
Elevage des petits ruminants en Kabylie, Algérie, et perspectives de développement
}

\author{
Khelaf Saidani ${ }^{1 *}$ Hocine Ziam ${ }^{1,2}$ Mourad Hamiroune ${ }^{3}$ \\ Souad Righi ${ }^{4}$ Ahmed Benakhla ${ }^{4}$
}

Mots-clés

Caprin, ovin, petits ruminants, production, reproduction, Algérie

Submitted: 28 May 2018

Accepted: 17 April 2019

Published: 12 July 2019

DOI: $10.19182 /$ remvt.31745

\begin{abstract}
Résumé
Une enquête a été menée pour étudier le système d'élevage, I'alimentation, la taille de l'exploitation, les principales races exploitées, certains paramètres de production et de reproduction, et des indicateurs socioéconomiques, notamment liés à la viabilité des exploitations, de 110 élevages ovins et caprins situés dans les wilayas de Bejaia et de Tizi Ouzou en Algérie, de juillet 2016 à janvier 2018. Les élevages étaient de type mixte, de petite taille (moins de 100 têtes) et à tendance viande. L'alimentation était basée sur les ressources sylvopastorales, le fourrage et la complémentation avec des concentrés. Une plus grande diversité de races a été rencontrée chez les ovins que chez les caprins. Par ailleurs, la race ovine Tazegzawt (récemment répertoriée et reconnaissable à ses taches noires à reflets bleuâtres d'où son nom en kabyle) était peu représentée, alors qu'il importerait de la préserver et de l'améliorer. Les petits ruminants étaient généralement abattus avant l'âge d'un an, très rarement après deux ans. Deux à quatre chevreaux ou agneaux naissaient par an. Les contraintes majeures étaient le manque de fourrage et la cherté des aliments, des fibres et des concentrés.
\end{abstract}

- Comment citer cet article : Saidani K., Ziam H., Hamiroune M., Righi S., Benakhla A., 2019. Small ruminant rearing in Kabylia, Algeria, and prospects for its development. Rev. Elev. Med. Vet. Pays Trop., 72 (2): 49-54, doi: 10.19182/remvt.31745

\section{INTRODUCTION}

L'élevage des petits ruminants est fortement ancré dans l'esprit de l'éleveur nord-africain (Rondia, 2006). En Algérie, l'élevage ovin constitue la colonne vertébrale de la production de viande rouge (Mebirouk-Boudechiche et al., 2014). Ainsi, la consommation de viande ovine est nettement supérieure à celle des bovidés, soit 10,512,0 kilogrammes par an par habitant (Alary et Boutonnet, 2006).

\footnotetext{
1. Institut des sciences vétérinaires, Université Blida 1, BP 270, route de Soomâa,
Blida, Algérie.

1. Institut des sciences vétérinaires, Université Blida 1, BP 270, route de Soomâa,
Blida, Algérie.

2. Laboratoire de biotechnologies, environnement et santé, Université Saad Dahlab Blida-1, Blida, Algérie.

3. Département des sciences agro-vétérinaires, Faculté des sciences de la nature et de la vie, Université Ziane Achour Djelfa, Djelfa, Algérie.

4. Département des sciences vétérinaires, Faculté des sciences de la nature et de la vie, Université El-Taref, Algérie.

* Auteur pour la correspondance Email : kamel_khelaf@yahoo.fr
}

Selon Djaout et al. (2017), le nombre d'ovins s'élève à 20688000 têtes avec neuf races génétiquement bien identifiées : Ouled Djellal, Rembi, Hamra, Berbère, Barbarine, D’Man, Sidaou, Tâadmit et Tazegzawt. Ces races locales ayant évolué, depuis l'antiquité, dans les conditions agroécologiques algériennes (steppiques et sahariennes) présentent une excellente adaptabilité basée sur la résilience, la prolificité, et la production de viande, de lait et de laine (Djaout et al., 2017). A ces races plus ou moins bien définies s'ajoutent plusieurs autres races et populations locales qui demeurent méconnues (Madani et al., 2015).

L'élevage caprin, d'environ 4,5 millions de têtes (Mouhous et al., 2013), est très répandu. Au nord il est cantonné aux zones montagneuses, mais l'essentiel de l'effectif est réparti dans les zones steppiques et subdésertiques (Moustaria, 2008). La population caprine locale, présente essentiellement en régions difficiles (montagnes, forêts, steppes et Sahara) et conduite en élevage extensif, valorise des ressources alimentaires pauvres pour produire de la viande (Madani et al., 2015). Etant appréciée comme viande rouge maigre, elle est très recherchée par les personnes atteintes de dyslipidémie ou à titre préventif contre l'obésité, le diabète de type 2 ou les troubles cardiovasculaires, en raison de sa faible teneur en graisse et sa richesse en 
muscles (Webb et al., 2005). Le lait de chèvre, avec sa haute valeur nutritive, est devenu dans certaines régions (Escareño et al., 2013), y compris la Kabylie, le succédané du lait de femme pour allaiter les bébés (Park, 2012 ; Sahraoui et al., 2016).

En Algérie, particulièrement dans les zones montagneuses, à l'instar des pays de l'Afrique du Nord et du Sahel, les petits ruminants contribuent substantiellement à la sécurité alimentaire, à l'économie des ménages montagnards, à leur maintien en territoires pauvres et peu accessibles, et rendent nécessaire une présence humaine dans des régions exposées à l'exode rural (Alary et al., 2011 ; Bengoumi et al., 2013 ; Madani et al., 2015).

Le présent travail avait pour objectif l'étude de quelques aspects de l'élevage des petits ruminants dans deux wilayas de la Kabylie : caractériser les élevages ovins et caprins, décrire les bâtiments et l'alimentation, recenser les races présentes, répertorier les races marginales et méconnues, évaluer les performances de production (viande et lait) et de reproduction (prolificité), recenser les pathologies dominantes et les moyens de lutte contre celles-ci, et aborder quelques aspects socioéconomiques de l'élevage des petits ruminants.

\section{MATERIEL ET METHODES}

\section{Région de l'étude}

Les deux wilayas du nord de l'Algérie où s'est déroulée l'enquête, en l'occurrence Béjaia ( $36^{\circ} 45^{\prime} \mathrm{N}$ et $5^{\circ} 04^{\prime}$ E) et Tizi Ouzou (36 $46^{\circ} \mathrm{N}$ et $4^{\circ} 03^{\prime} \mathrm{E}$ ), appartiennent à l'Atlas tellien, un ensemble de massifs montagneux côtiers.

\section{Déroulement de l'enquête}

Cette entreprise a été facilitée par les directions des services vétérinaires des deux wilayas et les subdivisions agricoles qui en dépendent et a été complétée par des visites de terrain, y compris sur le lieu de pâturage. Cependant, pour éviter de biaiser l'échantillonnage des + élevages de ruminants, des exploitations non recensées par les autorités agricoles ont également été incluses dans l'étude ; le seul critère d'exclusion a été la présence exclusive de bovins.

Une enquête à l'aide d'un questionnaire a été menée auprès des éleveurs de Bejaia et Tizi Ouzou, de juillet 2016 à janvier 2018. Au total 110 élevages ont été concernés. Afin de relever des indications sur les éleveurs, les ruminants et les écosystèmes, elle a porté sur la taille des élevages, les principales races exploitées, l'alimentation, la qualité des bâtiments d'élevage, la situation géographique des élevages selon le relief, les performances de production et de reproduction, l'identification des principales contraintes, le profil des éleveurs, les aspects socioéconomiques, la rentabilité et la viabilité des exploitations, le suivi sanitaire, et la lutte contre les pathologies majeures.

\section{Analyse des données}

Pour l'analyse des résultats, les indicateurs précédents ont été considérés comme les variables (ou facteurs). La méthode pour le dépouillement des données a été l'analyse factorielle des correspondances multiples (Baccini, 2010). Les différents types du test du chi deux ont été utilisés pour comparer des distributions entre elles (proportions ou pourcentages) ainsi que pour explorer une éventuelle association entre deux variables qualitatives.

Les statistiques descriptives ont été exécutées par le tableur Microsoft Excel 2010 alors que les tests d'hypothèse et l'analyse factorielle ont été réalisés par le logiciel open source R (2017) (version 3.3.3.). La librairie Facto Miner du logiciel R (R Core Team 2017) a servi à la réalisation de l'analyse des correspondances multiples (Lemercier et al., 2010).

\section{RESULTATS}

Le tableau I montre que les éleveurs en Kabylie combinaient assez souvent l'élevage des ovins à celui des caprins, beaucoup plus rarement petits ruminants et bovins $\left(\chi^{2}=66,3\right.$ et $\left.p=6,05 \times 10^{-13}\right)$.

Que l'élevage ait été caprin, ovin ou combiné à d'autres espèces, l'effectif dépassait rarement soixante têtes $\left(\chi^{2}=99,3\right.$ et $\left.p<2,20 \times 10^{-16}\right)$ (moins de $9 \%$ d'entre eux) ; un seul éleveur possédait plus de 100 têtes $(<1 \%)$.

Le tableau II montre que l'alimentation du cheptel était principalement basée sur le pâturage et, pour plus de la moitié d'entre eux, le pastoralisme sylvestre ; $77 \%$ des éleveurs complétaient par la distribution d'aliments concentrés $\left(\chi^{2}=102\right.$ et $\left.p<2,20 \times 10^{-16}\right)$; étant donné la qualité en général médiocre des végétaux disponibles, cette complémentation s'imposait surtout pour les femelles en production et les animaux d'engraissement, en particulier chez l'espèce ovine. Le complément alimentaire était généralement de l'aliment composé pour vaches laitières ou du pain sec broyé. Seulement $10 \%$ des éleveurs donnaient du foin à leurs petits ruminants, car les éleveurs kabyles préfèrent donner cet aliment aux bovins comme source de fibres alimentaires en prévention des troubles métaboliques.

La plupart des bâtiments d'élevage $\left(\chi^{2}=31,6\right.$ et $\left.p=2,27 \times 10^{-6}\right)$ étaient de type traditionnel, des bergeries intégrées dans les habitations des

\section{Tableau I}

Espèces et tailles des élevages dans deux wilayas de Kabylie en Algérie (juil. 2016 - janv. 2018) (n = 110)

\begin{tabular}{lcc|c|}
\hline Espèce & $\begin{array}{c}\text { Nb. } \\
\text { d'élevages }\end{array}$ & $\begin{array}{c}\text { Nb. } \\
\text { de têtes }\end{array}$ & $\begin{array}{c}\text { Nb. } \\
\text { d'élevages }\end{array}$ \\
\hline Ovin uniquement & 37 & $<20$ & 17 \\
Caprin uniquement & 29 & $20-39$ & 61 \\
Ovin et caprin & 31 & $40-60$ & 23 \\
Caprin et bovin & 4 & $61-100$ & 8 \\
Ovin et bovin & 6 & $>100$ & 1 \\
Bovin, caprin et ovin & 3 & & \\
\hline \multicolumn{1}{|c|}{$P=6,05 \times 10^{-13}$} & & $P<2,20 \times 10^{-16}$ \\
\hline
\end{tabular}

\section{Tableau II}

Alimentation et type de bâtiments dans deux wilayas de Kabylie en Algérie (juil. 2016 - janv. 2018) ( $n=110$ )

\begin{tabular}{|c|c|c|c|}
\hline \multicolumn{2}{|l|}{ Bâtiment d'élevage } & \multicolumn{2}{|l|}{ Alimentation } \\
\hline $\begin{array}{l}\text { Bergerie } \\
\text { bien aménagée }\end{array}$ & 7 & $\begin{array}{l}\text { Fourrage vert (herbe et } \\
\text { autres végétaux verts) }\end{array}$ & 15 \\
\hline $\begin{array}{l}\text { Bergerie } \\
\text { non équipée }\end{array}$ & 16 & $\begin{array}{l}\text { Fourrage sec (foin et paille) } \\
\text { et concentrés }\end{array}$ & 11 \\
\hline $\begin{array}{l}\text { Garage initialement } \\
\text { pour véhicules }\end{array}$ & 29 & $\begin{array}{l}\text { Fourrage en bergerie } \\
\text { et concentrés }\end{array}$ & 7 \\
\hline $\begin{array}{l}\text { Vieilles maisons } \\
\text { désaffectées }\end{array}$ & 41 & $\begin{array}{l}\text { Sylvopastoralisme } \\
\text { et concentrés }\end{array}$ & 67 \\
\hline $\begin{array}{l}\text { Habitat destiné } \\
\text { à d'autres animaux }\end{array}$ & 17 & $\begin{array}{l}\text { Sylvopastoralisme } \\
\text { exclusif }\end{array}$ & 18 \\
\hline $\mathrm{P}<2,27 \times 10^{-6}$ & & $P=2,20 \times 10^{-16}$ & \\
\hline
\end{tabular}


éleveurs, des garages ou de vieilles habitations aménagées. Le bâtiment adéquat et équipé n'était présent que chez sept éleveurs (6\%). Les autres types de bâtiments rencontrés étaient en général de vieilles bâtisses désaffectées ou des bâtiments simples en bois ou en tôle (tableau II).

Plus de $66 \%$ des éleveurs $\left(\chi^{2}=88,2\right.$ et $\left.p<2,20 \times 10^{-16}\right)$ exerçaient une autre activité, fonction libérale ou publique, seulement $20 \%$ étaient des éleveurs de petits ruminants professionnels, $12 \%$ étaient des éleveurs-agriculteurs, et environ $5 \%$ étaient des amateurs. (tableau III).

Parmi les élevages, seulement cinq étaient destinés à la vente de lait pour couvrir les dépenses d'exploitation. La plupart $\left(\chi^{2}=42,7\right.$ et $\mathrm{p}=1,18 \times 10^{-8}$ ) étaient des élevages mixtes ou orientés vers la production de viande, ou des élevages temporaires (commerce, achat et vente, engraissement de courte durée à l'approche des fêtes religieuses) (tableau III).

Pour les performances de production et de reproduction des petits ruminants, l'âge de début de la première gestation ne dépassait généralement pas un an. Les mises bas avaient lieu deux fois par an dans la plupart des élevages, au début du printemps et en automne, très rarement en été ou en hiver, étant donné qu'il n'y avait jamais d'induction des chaleurs ni de synchronisation des chaleurs. Le nombre de petits par femelle par an était de deux à quatre agneaux ou chevreaux. L'âge d'abattage ou de vente dépassait rarement 12 mois. Le poids à l'abattage ou à la vente était presque toujours inférieur à 40 kilogrammes pour les ovins, et inférieur à 25 kilogrammes pour les caprins. La quantité de lait de chèvre produite par jour ne dépassait jamais deux litres. Ainsi, le lait, dans le cas de tous les élevages enquêtés, couvrait à peine les besoins des familles des éleveurs. Il n'y avait pas de

\section{Tableau III}

Eleveurs et destination des élevages dans deux wilayas de Kabylie en Algérie (juil. 2016 - janv. 2018) (n = 110)

\begin{tabular}{|c|c|c|c|}
\hline \multicolumn{2}{|l|}{ Eleveur } & \multicolumn{2}{|c|}{ Destination de l'élevage } \\
\hline Professionnel & 22 & $\begin{array}{l}\text { Lait et vente } \\
\text { des animaux }\end{array}$ & 5 \\
\hline Eleveur agriculteur & 13 & Engraissement & 23 \\
\hline Eleveur et autre activité & 69 & Lait et viande & 42 \\
\hline \multirow[t]{2}{*}{ Amateur } & 6 & $\begin{array}{l}\text { Commerce } \\
\text { (achat et revente) }\end{array}$ & 31 \\
\hline & & $\begin{array}{l}\text { Couvrir les besoins } \\
\text { des familles }\end{array}$ & 9 \\
\hline \multicolumn{2}{|c|}{$P<2,20 \times 10^{-16}$} & \multicolumn{2}{|c|}{$P=1,18 \times 10^{-8}$} \\
\hline
\end{tabular}

\section{Tableau IV}

Répartition des races des petits ruminants dans deux wilayas de Kabylie en Algérie (juil. 2016 - janv. 2018)

\begin{tabular}{|c|c|c|c|}
\hline \multicolumn{2}{|l|}{ Ovins } & \multicolumn{2}{|l|}{ Caprins } \\
\hline Barbarin & 2 & Kabyle & 23 \\
\hline Berbère & 10 & Alpin & 8 \\
\hline Hamra & 11 & Saanen & 19 \\
\hline Ouled Djellal & 29 & Mancha & 5 \\
\hline Rembi & 16 & & \\
\hline Tazegzawt & 3 & Croisé & 12 \\
\hline Croisé & 6 & & \\
\hline \multicolumn{2}{|c|}{$P=1,65 \times 10^{-8}$} & \multicolumn{2}{|c|}{$P=0,00211$} \\
\hline
\end{tabular}

production laitière destinée aux fromageries dans le contexte de notre étude. La production de presque tous les élevages était mixte mais avec une tendance aux animaux à viande.

Le mode de reproduction était la monte libre ; les boucs étaient en permanence avec les chèvres et les béliers avec les brebis. La monte contrôlée n'était pas pratiquée par les éleveurs enquêtés et l'insémination artificielle était inexistante.

La race ovine arabe blanche Ouled Djellal était prédominante $\left(\chi^{2}=47,3\right.$ et $\left.p=1,65 \times 10^{-8}\right)$ et la race caprine kabyle $\left(\chi^{2}=16,8\right.$ et $\mathrm{p}=0,00211$ ) était la plus représentée (tableau IV).

L'analyse factorielle des correspondances multiples (figures 1 et 2) a permis de déduire que les caprins étaient plus adaptés aux régions montagneuses ; les exploitations caprines étaient rares dans les zones périurbaines (tableau V). Inversement, les ovins étaient plus présents en plaine, étant moins adaptés aux régions difficiles, montagnes et collines. En effet, l'application du test de chi deux d'indépendance a montré une liaison très significative entre l'implantation de l'élevage et l'espèce de petits ruminants élevée $\left(\chi^{2}=28,7\right.$ et $\left.p=2,57 \times 10^{-6}\right)$. Aucun des éleveurs ne possédait de grandes surfaces agricoles permettant les cultures fourragères. Leurs terrains étaient étroits et seulement adaptés aux cultures maraîchères, ce qui expliquait la petite taille des élevages. Les petits ruminants étaient généralement élevés en semi-extensif

Les pathologies majeures sont reportées dans le tableau VI. Les maladies épizootiques plus graves comme la fièvre aphteuse et la fièvre catarrhale étaient plus rares.

\section{Tableau V}

Répartition des élevages selon l'espèce animale et localisation dans deux wilayas de Kabylie en Algérie (juil. 2016 - janv. 2018)

\begin{tabular}{lcc} 
Situation & Elevage ovin & Elevage caprin \\
\hline Périurbain & 17 & 5 \\
Plaine & 31 & 11 \\
Colline & 19 & 17 \\
Montagne & 10 & 34
\end{tabular}

\section{Tableau VI}

Pathologies dominantes dans les élevages de deux wilayas de Kabylie en Algérie (juil. 2016 - janv. 2018)

$$
(\mathrm{n}=110)
$$

\begin{tabular}{lc} 
Pathologies & Nb. d'élevages \\
\hline Bronchopneumonie & 31 \\
Indigestion & 15 \\
Acidose métabolique & 13 \\
Météorisation & 10 \\
Diarrhées & 7 \\
Dystocies & 4 \\
Rétention placentaire & 2 \\
Autres pathologies & 1 \\
Aucune pathologie signalée & 27 \\
\hline \multicolumn{2}{c}{$P=2,401 \times 10^{-11}$} \\
\end{tabular}




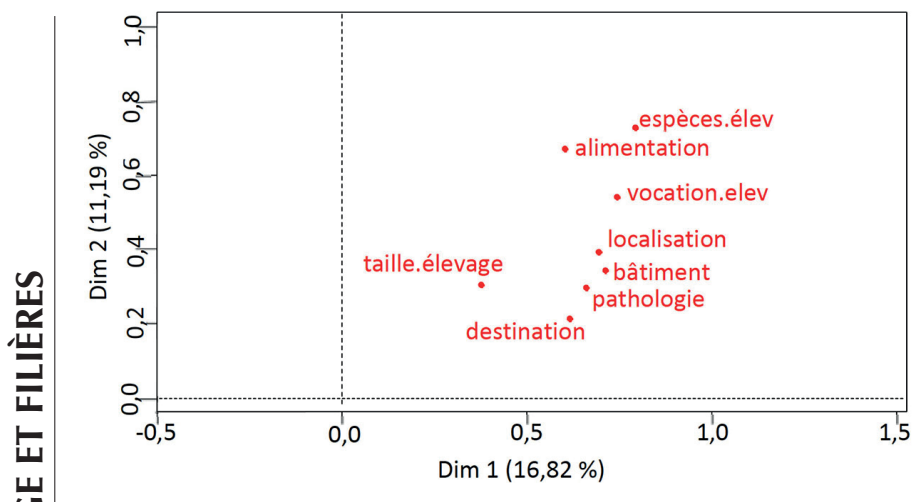

Figure 1 : résultats de l'analyse factorielle des correspondances multiples ; plan des variables : espèce animale élevée, alimentation, vocation de l'éleveur, localisation, taille de l'élevage (nombre total de têtes), type de bâtiment, pathologies dominantes, destination de l'élevage.

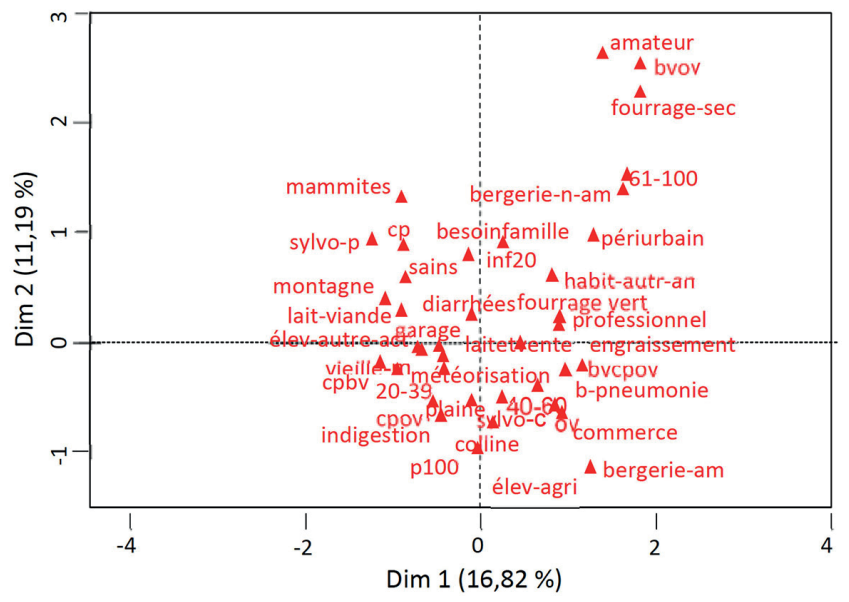

Figure 2 : résultats de l'analyse factorielle des correspondances multiples ; plan des modalités : bv (bovin), cp (caprin), ov (ovin), bergerie-am (bergerie aménagée), bergerie $n$-am (bergerie non aménagée, élev-agri (éleveur-agriculteur), élev-autre (éleveur et autre activité), besoinfamille (élevage pour besoins de famille), sylvo-p (sylvopastoralisme), inf20 (moins de 20 têtes dans l'élevage), p100 (plus de 100 têtes), vieille-m (vieilles maisons).

\section{DISCUSSION}

L'élevage des ovins est souvent combiné à celui des caprins, plus rarement les petits ruminants sont élevés avec les bovins. En fait, les ovins et les caprins sont considérés comme des espèces voisines de ruminants alors que le bovin fait partie du gros bétail. Similairement, dans d'autres régions algériennes, l'élevage caprin est souvent associé à celui des ovins (Sahraoui et al., 2016). Dans la plupart des élevages visités (61\%), il y avait des caprins. Dans les petits élevages caprins, des chèvres laitières étaient élevées pour couvrir les besoins familiaux en lait, surtout ceux des enfants et des nourrissons.

Concernant l'alimentation des petits ruminants, les éleveurs utilisaient la végétation naturelle assez abondante en Kabylie ; près de $50 \%$ de la wilaya de Tizi Ouzou était couverte de végétation spontanée (Kadi et al., 2016), graminées et légumineuses surtout, mais aussi chênes Zen et Afarez. Les concentrés étaient toujours achetés et non produits par l'éleveur en raison de l'exiguïté des surfaces agricoles utiles (Djermoun et Chehat, 2012).
Les bâtiments d'élevage étaient souvent précaires et insalubres, comme au Maroc où Alami et al. (2005) signalent des logements de troupeaux caprins construits en argile avec des toitures en tôle. De même, selon Pacheco (2006), au Portugal ces bâtiments sont généralement très anciens, peu fonctionnels, peu ventilés et peu éclairés. Ce type d'abri affecte la santé des animaux ; les maladies respiratoires sont fréquentes en raison de la mauvaise ventilation en hiver et des coups de chaleur en été, où la température peut atteindre $40{ }^{\circ} \mathrm{C}$, avec des périodes de sirocco très néfastes à l'élevage des animaux de rente dont les petits ruminants. En effet, la plupart des cas de bronchopneumonie étaient consécutifs à des périodes de vents chauds. A la différence de certaines régions steppiques (Smaali et Chammam, 2017), le lait de brebis n'était presque jamais utilisé pour l'alimentation humaine, il était uniquement destiné à l'allaitement des agneaux. Par conséquent, en Kabylie, les ovins sont élevés pour leur viande.

Six races ovines et quatre caprines étaient présentes dans la zone d'étude outre les produits de leur croisement, ce qui témoigne d'une grande diversité génétique, un élément-clé dans le développement durable (Djaout et al., 2017). Par ailleurs, la prédominance de la race ovine Ouled Djellal montre une fois de plus qu'elle est une race principale (Chekkal et al., 2015) qui continue de croître. Des races à faible effectif, comme la Tazegzawt (reconnaissable à ses taches noires à reflets bleuâtres, d'où son nom en kabyle), sont menacées et en danger d'extinction (Moulla et al., 2015) ; il faudrait donc la préserver en la soustrayant au croisement non contrôlé (Chekkal el al., 2015).

La production de fourrage était malheureusement sujette aux aléas climatiques. Ceci rejoint les résultats d'Arbouche (1995) et de Nedjraoui (2001) : « En Kabylie, les animaux sont nourris en hiver de feuilles de figuier et de rameaux d'olivier et au printemps ils sont conduits dans les champs en jachère qui leur fournissent une alimentation suffisante, puis dans les parties montagneuses sur les pacages estivaux. »

La période la plus difficile dans l'alimentation des ruminants s'étendait de juillet à mars. Par contre, le reste de l'année, les animaux profitaient au maximum des parcours naturels comme cela a été rapporté pour les régions steppiques : "L'alimentation des troupeaux dans la région est ainsi basée surtout sur les pâtures naturelles ; en général, lorsque la pluviométrie est suffisante pendant l'hiver précédent, la poussée de la végétation arrive à son maximum aux mois d'avril et de mai, par conséquent, les troupeaux profitent pleinement de cette végétation jusqu'en juillet » (Zouyed, 2005). En période de disette, les années où la pluviométrie est faible, à partir du mois d'août, les petits ruminants étaient maintenus en stabulation libre (non attachés) et nourris de foin, de paille, de feuilles d'arbustes fourragers (frêne, chêne-liège, orme, oléastre) comme cela a été rapporté par Kadi et al. (2016) pour les caprins. Laisser les ruminants profiter de la végétation naturelle constitue un atout majeur dans le contexte actuel d'augmentation du coût des matières premières, notamment des céréales (Mebirouk-Boudechiche et al., 2014). La contrainte majeure pour presque tous les élevages était le coût élevé de l'alimentation, le foin et surtout les concentrés. En effet, l'offre fourragère, que ce soit pour les bovins, les ovins ou les caprins, est insuffisante (Kadi et al., 2016).

Même si les dominantes pathologiques étaient les bronchopneumonies, les maladies métaboliques ou les troubles de la reproduction, des maladies épizootiques étaient déclarées chez les petits ruminants, comme la peste des petits ruminants, la fièvre aphteuse et la fièvre catarrhale du mouton, en accord avec les constats de Kardjadj (2017). Les systèmes d'élevage semi-extensif et extensif étaient dominants, et le système intensif a été récemment introduit, comme rapporté par Kardjadj et al. (2016).

Les ovins et rarement les caprins étaient vaccinés contre la clavelée et la fièvre aphteuse, et contre la brucellose avec des gouttes oculaires. Quelques élevages ovins bénéficiaient d'un traitement antiparasitaire, 
et l'ivermectine et l'albendazole étaient utilisés à titre préventif ou combinés à des antibiotiques ou des complexes vitaminés. Le vétérinaire n'intervenait presque jamais dans les élevages caprins. Ces résultats rejoignent ceux d'Arbouche (1995).

Les caprins étaient principalement élevés dans les régions montagneuses grâce aux parcours naturels notamment forestiers, leur lait était destiné à nourrir les familles des éleveurs, exceptionnellement à la vente ou la transformation artisanale. En revanche, les ovins étaient élevés dans les zones moins difficiles, comme les plaines, ils étaient principalement exploités pour leur viande et leur laine, comme dans les régions de grands élevages ovins (Yabrir et al., 2015). Le sylvopastoralisme était, de loin, moins pratiqué dans l'élevage ovin.

De grands efforts ont été déployés par les autorités algériennes pour tenter de résoudre les problématiques du secteur de l'élevage des animaux de rente et d'améliorer leur productivité, surtout depuis l'émergence de la nouvelle politique agricole du ministère de l'Agriculture et de Développement rural en 2000. Cependant, l'agriculture en général et l'élevage en particulier continuent de subir des contraintes d'ordre politique, social, écologique et environnemental. Parmi leurs effets citons a) les aléas climatiques, surtout la sécheresse et la faible pluviométrie qui pénalisent le profil fourrager notamment des parcours naturels, b) la faible maîtrise de la conduite des élevages, et des aspects de rationnement et de nutrition, c) les performances de production et de reproduction très faibles dans certains élevages en raison des troubles de la reproduction et des avortements parfois enzootiques, d) la présence de pathologies contagieuses, comme la brucellose et la fièvre aphteuse et autres pathologies, ou liées au manque d'hygiène, comme les mammites, les métrites, les avortements, les boiteries et les diarrhées, e) la diminution de la population active rurale au profit d'une surpopulation urbaine, f) la petite taille des exploitations, g) l'orientation des élevages vers l'engraissement des ovins et des bovins au détriment de la production laitière, h) l'absence d'élevages caprins à vocation laitière en dépit de la très haute valeur nutritionnelle du lait de chèvre comparé à d'autres laits, par exemple de vache, i) les besoins des familles d'éleveurs à peine couverts par la production laitière, j) l'absence d'action sanitaire à titre curatif et encore moins préventif chez les caprins, et k) l'administration aux ovins d'antimicrobiens à large spectre, associés à des anti-inflammatoires, sans diagnostic préalable, seulement sur la base des symptômes.

Les constats de « a » à «g » rejoignent ceux de Kabir (2015). Pour apporter des solutions, il faudrait promouvoir l'élevage caprin dans les régions montagneuses de la Kabylie, la chèvre étant très rustique et capable d'exploiter et de valoriser les végétaux les plus pauvres, quasi inutilisables par les ovins ou les bovins (Kadi et al., 2013 ; Mouhous et al., 2013). Le système d'élevage caprin en extensif, notamment de type laitier, n'a que de très faibles charges car la plus grande partie des dépenses sont inhérentes à l'achat des animaux, des fourrages secs (foin) et des concentrés (Mouhous et al., 2013). Par ailleurs, la situation actuelle caractérisée par une forte demande de produits caprins constitue une incitation pour l'émergence d'une filière lait caprin (Sahraoui et al., 2016), laquelle, par la même occasion, allégerait la pression de la demande en lait de vache. Enfin, il faudrait favoriser la viabilité des élevages en substituant des élevages de femelles reproductrices, allaitantes ou laitières aux parcs d'engraissement, qui constituent des élevages temporaires.

\section{CONCLUSION}

Pour pallier le déficit de fourrages, le facteur limitant le plus important, il faudrait développer la culture fourragère irriguée, et l'exploitation des parcours de brousses et de forêts par les caprins. Il faudrait également valoriser les sous-produits des cultures maraîchères et céréalières. Pour réduire l'exode des populations rurales actives, il serait nécessaire d'améliorer le cadre de vie de celles-ci et de prendre des mesures incitatives. Pour réduire les contraintes sanitaires et économiques liées à l'élevage, il devrait y avoir un accompagnement adéquat des éleveurs. En effet, l'Etat algérien a mis des moyens colossaux (Kabir 2015) pour stimuler la production animale (notamment la production laitière, surtout bovine), mais le point faible de cette politique volontariste (et sans doute la cause de résultats modestes) réside dans le manque d'accompagnement technique des éleveurs : seuls les vétérinaires de terrain assurent en partie ce rôle de conseil.

En définitive, une production laitière caprine pourra sans nul doute atténuer les déficits en lait imposant l'importation des poudres de lait. La viande caprine mérite sa réputation de viande salubre, moins riche en acides gras saturés ; elle est idéale dans la prévention des maladies cardiovasculaires. Pour les ovins, il faudrait favoriser le système d'élevage extensif pour réduire les charges de l'élevage et améliorer la qualité de la viande ovine étant donné que sa composition biochimique est déterminée par l'alimentation.

\section{Remerciements}

Les auteurs remercient l'ensemble du personnel des directions des services vétérinaires et les éleveurs des wilayas de Bejaia et Tizi Ouzou.

\section{Déclaration des contributions des auteurs}

$\mathrm{KS}$ et $\mathrm{HZ}$ ont participé à la conception de l'enquête, à la collecte des données et à la rédaction de la première version ; KS a effectué les analyses statistiques ; MH, SR et AB ont contribué à l'analyse, à l'interprétation des données et à la révision du manuscrit ; tous les auteurs ont contribué à la révision critique du manuscrit dont ils ont approuvé la forme actuelle.

\section{REFERENCES}

Alami N., Ben Bati M., Boukharta R., Jout J., Zahrou A., 2005. Quelle stratégie de recherche-développement pour l'élevage caprin dans la province de Chefchaouen, Maroc ? ICRA-INRA-DPA, Chefchaouen, Chambre d'agriculture, Conseil régional, Tétouan, Algérie, 74 p. (Sér. Doc. Travail ; 127)

Alary V., Boutonnet J.P., 2006. L'élevage ovin dans l'économie des pays du Maghreb. Un secteur en pleine évolution. Sécheresse, 17 (1-2) : 40-46

Alary V., Duteurtre G., Faye B., 2011. Elevages et sociétés : les rôles multiples de l'élevage dans les pays tropicaux. Prod. Anim., 24 (1) : 145-156

Arbouche F., 1995. Contribution à l'étude d'un facteur limitant le fonctionnement de la phytocénose : cas du pâturage dans la cédraie du Belzma (Aurès). Thèse Magister, INA, Algérie, 132 p.

Baccini A., 2010. Statistique descriptive multidimensionnelle (analyse factorielle). Institut de mathématiques, Toulouse, France

Bengoumi M., Ameziane El Hassani T., 2013. Evolution and efficacy of transfer of technologies in small ruminant production systems in North Africa. In: $8^{\text {th }}$ Int. Seminar FAO-CIHEAM network on sheep and goat technology creation and transfer in small ruminants: roles of research, development services and farmer associations, Tangier, Morocco, 11-13 June 2013. Options Méditerr. Sér. A: 15-24

Chekkal F., Benguega Z., Meradi S., Berredjouh D., Boudibi S., Lakhdari F., 2015. Guide de caractérisation phénotypique des races ovines de l'Algérie. CRSTRA, Biskra, Algérie

Djaout A., Afri-Bouzebda F., Chekal F., El-Bouyahiaoui R., Rabhi A., Boubekeur A., Benidir M., et al., 2017. Etat de la biodiversité des « races » ovines algériennes. Genet. Biodivers. J., 1: 11-26

Djermoun A., Chehat F., 2012. Le développement de la filière lait en Algérie : de I'autosuffisance à la dépendance. Livest. Res. Rural Dev., 24, 22

Escareño L., Salinas-Gonzalez H., Wurzinger M., Iñiguez L., Sölkner J., MezaHerrera C., 2013. Dairy goat production systems. Status quo, perspectives and challenges. Trop. Anim. Health Prod., 45: 17-34, doi: 10.1007/ s11250-012-0246-6 
Kabir A., 2015. Contraintes de la production laitière en Algérie et évaluation de la qualité du lait dans l'industrie laitière (constats et perspectives). Thèse Doct., Faculté des sciences de la nature et de la vie, Université d'Oran 1, Algérie

Kadi S.A., Djellal F., Hassini F., Mouhous A., 2016. Pratiques alimentaires dans les élevages caprins dans la région montagneuse de Tizi-Ouzou en Algérie. In: The value chains of Mediterranean sheep and goat products. Organisation of the industry, marketing strategies, feeding and production systems (Eds. Napoléone M., et al.). Options Méditerr. Sér. A. (115)

Kadi S.A., Hassini F., Lounas N., Mouhous A., 2013. Caractérisation de l'élevage caprin dans la région montagneuse de Kabylie en Algérie. In: $8^{\text {th }}$ Int. Semin. FAO-CIHEAM network on sheep and goat technology creation and transfer in small ruminants: roles of research, development services and farmer associations, Tangier, Morocco, 11-13 June 2013. Options Méditerr. Sér. A (108): 451-456

Kardjadj M., 2017. An epidemiological overview of small ruminant diseases in Algeria. Rev. Sci. Tech. Off. Int. Epizoot., 36 (3)

Kardjadj M., Kouidri B., Metref D., Luka P.D., Ben-Mahdi M.H., 2016. Abortion and various associated risk factors in small ruminants in Algeria. Prev. Vet. Med., 123: 97-101, doi: 10.1016/j.prevetmed.2015.11.015

Lemercier C., Milani P., Sofio S., 2010. Tutoriel FactoMineR pour l'analyse des correspondances multiples avec une petite annexe sur la classification automatique. www.quanti.ihmc.ens.fr/IMG/pdf/Tutoriel_FactomineR_ACM.pdf

Madani T., Sahraoui H., Benmakhlouf H., 2015. Elevage caprin en Algérie : Systèmes d'élevage, performances et mutations. In : Workshop Natl. Valorisation des « races » locales ovines et caprines à faibles effectifs, un réservoir de diversité génétique pour le développement local, INRAA, Alger, 2-3 mars 2015

Mebirouk-Boudechiche L., Boudechiche L., Ferhat R., Tahar A.., 2014. Relation entre disponibilité en herbe, ingestion et activité de béliers au pâturage. Arch. Zootec., 63 (242) : 277-287, doi : 10.4321/S0004-05922014000200006

Mouhous A., Bouraine N., Bouaraba F., 2013. L'élevage caprin en zone de montagne. Cas de la région de Tizi-Ouzou (Algérie). Rencontres Rech. Rumin. : 20

\section{Summary}

Saidani K., Ziam H., Hamiroune M., Righi S., Benakhla A. Small ruminant rearing in Kabylia, Algeria, and prospects for its development

A survey was conducted to study the livestock system, feeding, farm size, main breeds reared, some production and reproduction parameters, and socioeconomic indicators, related in particular to farm viability, of 110 sheep and goat farms located in the wilayas of Bejaia and Tizi Ouzou in Algeria, from July 2016 to January 2018. The farms were of a mixed type, small-sized (less than 100 head) and meat-oriented. Feeding was based on silvopastoral resources, fodder, and supplementation with concentrates. A higher diversity of breeds was found in sheep than in goats. In addition, the Tazegzawt sheep breed (recently referenced and recognizable by its black spots with bluish hues, hence its Kabyle name) was little represented, although it would be important to preserve and improve it. Small ruminants were generally slaughtered before the age of one year, very rarely after two years. Two to four kids or lambs were born per year. The main constraints were the lack of fodder and the high cost of feed, fiber and concentrates.

Keywords: goats, sheep, small ruminants, production, reproduction, Algeria
Moulla F., El-Bouyahiaoui R., 2015. Populations ovines locales algériennes de la Kabylie : Ressources génétiques animales méconnues et en danger d'extinction. In : Workshop Natl. Valorisation des « races » locales ovines et caprines à faibles effectifs, un réservoir de diversité génétique pour le développement local, INRAA, Alger, 2-3 mars 2015

Moustaria A., 2008. Identification des races caprines des zones arides en Algérie. Rev. Rég. Arid., 21 : 1378-1382

Nedjraoui D., 2003. Profil fourrager de I'Algérie. FAO, Rome, Italie

Pacheco F., 2006. Les systèmes d'élevage laitier dans la région de l'Entre Douro e Minho : Réflexions sur un dispositif d'appui technique aux éleveurs. Options Méditerr. Sér. A. (70) : 179-186

Park Y.W., 2012. Goat milk and human nutrition. In: Proc. 1st Asia Dairy Goat Conf., Kuala Lumpur, Malaysia, 9-12 Apr. 2012

R Core Team, 2017. A language and environment for statistical computing. $R$ Foundation for Statistical Computing, Vienna, Austria

Rondia P., 2006. Aperçu de l'élevage ovin en Afrique du Nord. Filière Ovine Caprine (18) : 11-14

Sahraoui H., Madani T., Kermouche F., 2016. Le développement d'une filière lait caprin en régions de montagne : un atout pour un développement régional durable en Algérie. In: The value chains of Mediterranean sheep and goat products. Organisation of the industry, marketing strategies, feeding and production systems (Eds. Napoléone M., et al.). Options Méditerr. Sér. A. (115)

Smaali S., Chemmam M., 2017. Potentiel de production laitière de brebis primipares Ouled Djellal en système extensif amélioré (reproduction, suralimentation). Livest. Res. Rural Dev., 29, 179 (consulté 1 avr. 2018)

Webb E.C., Casey N.H., Simela I., 2005. Goat meat quality. Small Rumin. Res., 60: 153-166, doi: 10.1016/j.smallrumres.2005.06.009

Yabrir B., Laoun A., Chenouf N.S., Mati A., 2015. Caractéristiques des élevages ovins de la steppe centrale de l'Algérie en relation avec l'aridité du milieu : cas de la wilaya de Djelfa. Livest. Res. Rural Dev., 27, 207 (consulté 27 mars 2018)

Zouyed I., 2005. Engraissement des ovins : caractéristiques des carcasses et modèles de classification. Mém. Magister pathologies des ruminants, Université Constantine, $87 \mathrm{p}$.

\section{Resumen}

Saidani K., Ziam H., Hamiroune M., Righi S., Benakhla A. Cría de pequeños rumiantes en Cabilia, Argelia, y prospectos para su desarrollo

Se llevó a cabo una encuesta para estudiar el sistema de cría, alimentación, tamaño de la finca, principales razas criadas, algunos parámetros de producción y reproducción, e indicadores socioeconómicos, relacionados en particular con la viabilidad de las fincas, en 110 fincas de ovejas y cabras localizadas en las divisiones administrativas de Behaia y Tizi Ouzou, en Argelia, entre julio 2016 y enero 2018. Las fincas eran de tipo mixto, de pequeño tamaño (menos de 100 cabezas) y orientadas hacia carne. La alimentación se basó en recursos silvopastorales, forraje y suplementos con concentrados. Se encontró una mayor diversidad de razas en ovejas que en cabras. Además, la raza ovina de Tazegzawt (recientemente referenciada y reconocible por sus manchas negras con tonos azulados, lo que explica su nombre en cabilio) se vio poco representada, a pesar de que sería importante preservarla y mejorarla. Los pequeños rumiantes fueron generalmente sacrificados antes de un año de edad, rara vez después de los dos años. Nacieron de dos a cuatro cabritos por año. Los principales obstáculos fueron la falta de forraje y el alto costo del alimento, fibras y concentrados.

Palabras clave: caprino, ovino, pequeños rumiantes, producción, reproducción, Argelia 